\title{
Calculation of improved features of distributed-feedback tapered master-oscillator power-amplifiers
}

\author{
V. Tronciu ${ }^{1}$, M. Lichtner ${ }^{1}$, M. Radziunas ${ }^{1}$, U. Bandelow ${ }^{1}$, H. Wenzel ${ }^{2}$ \\ ${ }^{1}$ Weierstrass Institute for Applied Analysis and Stochastics Mohrenstr. 39, 10117 Berlin, Germany \\ ${ }^{2}$ Ferdinand Braun Institut für Höchstfrequenztechnik, Gustav-Kirchhoff-Str. 4, 12489 Berlin, Germany
}

\begin{abstract}
During recent years, compact lasers emitting single-frequency, diffraction limited continuous-wave CW beams at an optical power of several Watts have received considerable attention regarding several applications, such as frequency conversion, free-space communications, and pumping of fiber lasers and fiber amplifiers. A device which is capable to maintain a good beam quality and wavelength stability in the Watt range is the monolithically integrated master-oscillator power-amplifier (MOPA), where either a distributed Bragg reflector (DBR) [1] or a distributed feedback (DFB) [2] laser and a flared (or tapered) gain-region amplifier are combined on a single chip. Figure 1 shows the structure of the DFB MOPA device that has been considered. It consists of an index guided DFB ridge-waveguide (RW) laser and a gain-guided tapered amplifier. Both regions are separately contacted and can be driven independently. The PA has a total flare angle of $6^{\circ}$. In order to understand the origin of different instabilities and transitions between different states we analyze and simulate a mathematical model based on travelling wave equations for the complex slowly varying amplitudes of the counterpropagating optical fields coupled to a diffusion equation for the excess carrier density and an equation for gain dispersion [2, 3]. Figure 2 (left) shows calculated optical spectra with increasing PA injection currents. The diagrams show a basic red shift of the lasing wavelength with increasing injected current as well as periodic changes of the lasing modes. To understand the properties of the laser, we consider the laser dynamics in terms of bifurcation diagrams. We perform a bifurcation analysis by using the power amplifier current as bifurcation parameter. We find Hopf bifurcation points and a scenario compatible with the quasiperiodic route to chaos is obtained as the injection current is increased.

In the subsequent analysis, we consider the influence of the coupling coefficient on the output characteristics of DFB MOPA. When the coupling coefficient is slightly increased from $250 \mathrm{~m}^{-1}$ the regions of instabilities become smaller until they disappear at higher values where the laser operates at stable $\mathrm{CW}$ state, which denote a better laser performance (see right panel of Fig.2). We mention that for the high values of coupling coefficient the backward field intensity is decreased. Moreover, in case of high coupling the high intensity of forward field is concentrated in the centre of DFB section compared to low coupling where the intensity maxima are located at the borders. An alternative approach to obtain better output characteristics of the DFB MOPA is to use a quarter wavelength shifted grating. We predict theoretically that when such a grating is introduced under high coupling coefficient the laser is operating in more stable regime compared with that of a laser with normal grating. We believe that our work provides a good basis for future study, and, in particular, provides some pointers for more detailed investigations of mechanisms behind the MOPA effects.
\end{abstract}

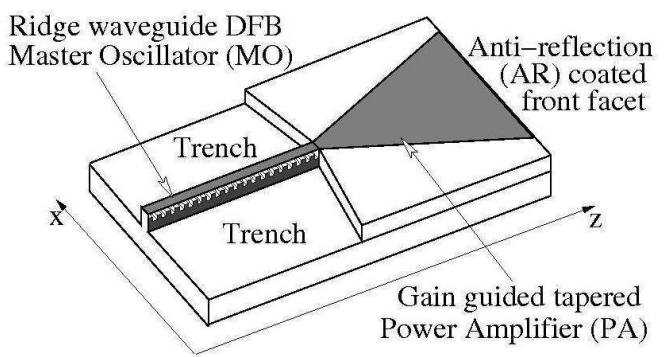

Fig. 1 Left: Schematic view of DFB MOPA.
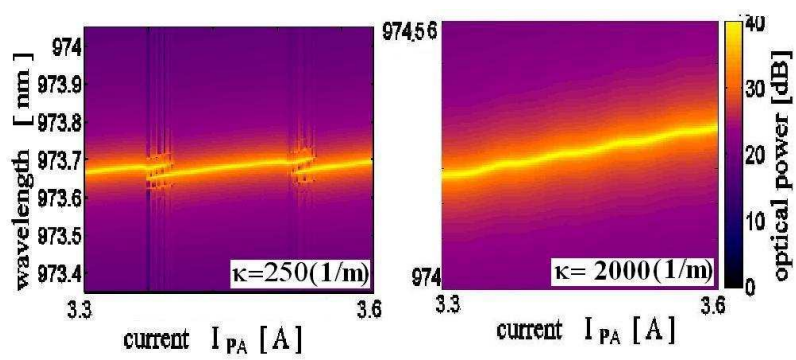

Fig.2 Mapping of the calculated optical spectra for different values of coupling coefficient and a fixed MO injected current $I_{M O}=0.35 \mathrm{~A}$.

\section{References}

[1] H. Wenzel, K. Paschke, O. Brox, F. Bugge, A. G. J. Fricke, A. Knauer, P. Ressel, and G. Erbert, "10-W continuous-wave monolithically integrated master-oscillator power-amplifier," Electron. Lett., vol. 43, no. 3, pp. 160-161, Feb. 2007.

[2] M. Spreemann, M. Lichtner, M. Radziunas, U. Bandelow, and H. Wenzel "Measurement and simulation of distributed-feedback tapered master oscillators power-amplifiers. Accepted IEEE J. Quant. Electron. (2009)

[3] A. Egan, C.Z.Ning, J.V. Moloney, R.A. Indik,M.W. Wright, D.J. Bossert, J.G. McInerney, "Dynamic instabilities in master oscillator power amplifier semiconductor lasers", IEEE J. Quant. Electron., 34(1), 166-170 (1998) 